\title{
T2.O.M. T.O.M.: Techniques and Technologies for an Ontology-Based Mobility Tool with Open Maps
}

\author{
Michele Ruta, Floriano Scioscia, Saverio Ieva, and Eugenio Di Sciascio \\ Politecnico di Bari, \\ via Re David 200, I-70125, \\ Bari, Italy \\ \{m.ruta,f.scioscia, disciascio\}@poliba.it ieva@deemail.poliba.it
}

\begin{abstract}
Nowadays, vehicle navigation systems have a limited effectiveness in satisfying user interests and needs. They basically locate the user's GPS position on a map and calculate a path toward a given destination. This paper presents an innovative semantic-based navigation system, acting as intelligent agent offering an advanced assistance during a road trip. It integrates a mobile matchmaker, which allows a knowledgeoriented destination discovery, in order to fully accomplish requirements of a user during her travel. The framework we propose is based on open standards, and particularly it uses crowd-sourced maps available under the OpenStreetMap project, where Points Of Interest (POI) of the basic cartography are semantically featured in order to enable novel services supporting a traveling user.
\end{abstract}

\section{Introduction}

Road congestion, environmental pollution and uncontrolled expenditure of energetic resources are increasingly urgent problems in many urban areas. In the last few years, the application of ICT to the transportation domain has opened promising possibilities to enable smarter traffic management solutions, while also promoting new services for drivers and passengers. This world-wide effort is usually referred to ITS (Intelligent Transport System) initiative. ITS aims at improving travellers' safety and satisfaction, as well as reducing traffic and transportation times and costs. In the short to medium run, the best that could be reasonably achieved is a combination of (i) automated traffic surveillance and road charging in congested areas with (ii) a set of assistive measures exploiting information and communication technologies within vehicular equipments.

Particularly, assisted navigation systems support drivers in planning trips and during travels: they are up to now one of the most widespread parts of ITS technological effort. Navigation softwares allow to locate and track a user, to calculate a route toward a given destination and enrich the travelling experience through visual maps and voice guidance. Nevertheless, current navigators present several limits. First of all they calculate a path by using minimum distance criteria without taking into account factors influencing travel such as road

F. Daniel and F.M. Facca (Eds.): ICWE 2010 Workshops, LNCS 6385, pp. 199-210, 2010.

(C) Springer-Verlag Berlin Heidelberg 2010 
congestions, accidents, dangerous routes due to either weather conditions and vehicle state. Furthermore, the informative content supporting map navigation is currently very low, as the only advanced search option they enable is based on POI (Point Of Interest). Unfortunately a POI can be identified and selected only through a name-based or category-based search, filtered according to proximity criteria. Such syntactic-based resource retrieval is inherently affected by poor recall, since only exact matches are supported. Moreover, the user cannot indicate characteristics and properties the destination should have, so that her personal preferences and collateral requirements conditioning path calculation are not taken into account.

This paper introduces an innovative framework to enhance functionalities of navigation systems for better assisting users in their trips, envisioning a general approach to retrieve and provide information which may be useful not only to enhance travel satisfaction and safety, but also to regulate vehicular traffic and environmental impact. Borrowing from the Semantic Web initiative, we propose a knowledge-based system whose final goal is to provide a navigation based on semantics of both user's needs and profile and on context annotation. Knowledge Representation theoretical studies are adapted and applied to ITS field. This opens new interesting possibilities, including: formalization of vehicles, context and actors annotations that become machine understandable so enabling interoperability; reasoning on descriptions and inference of new knowledge; Open World Assumption (OWA) by-passing structured data models. By means of formal ontologies, expressed using Description Logics (DLs) formalisms [1] and particularly OWI 1 , knowledge about transportation and travel domain can be modeled and exploited in order to derive new information from the one stated within metadata associated to POIs and user profiles.

Previous framework and approach has been implemented in an open source mobile navigator equipped with deductive reasoning capabilities, able to leverage semantically enriched crowd-sourced maps available under the OpenStreetMap project2. POI annotation enables a logic-based discovery of location features w.r.t. an articulate request expressed by the user. Based on the formal semantics of such descriptions, POIs are ranked according to the degree of satisfaction of the request. A lightweight version of non-standard inference algorithms, i.e., Concept Abduction, Concept Contraction and Bonus Calculation [3] is used for that. Furthermore, such an approach allows to provide the user with an explanation of matchmaking outcomes, in order to increase her confidence in the quality of search results and to foster further interactions with the system.

The remaining of the paper is structured as follows: in the next section, current state of the art in ITS and navigation systems is briefly recalled, Section 3 outlines system features and architecture evidencing its peculiarities and in Section 4 a toy case study will explain the proposed approach and the rationale motivating it. Finally conclusion and future work terminate the paper.

${ }^{1}$ OWL 2 Web Ontology Language overview, W3C Recommendation 27 October 2009, http://www.w3.org/TR/2009/REC-owl2-overview-20091027/

2 OpenStreetMap: http://www.openstreetmap.org/ 


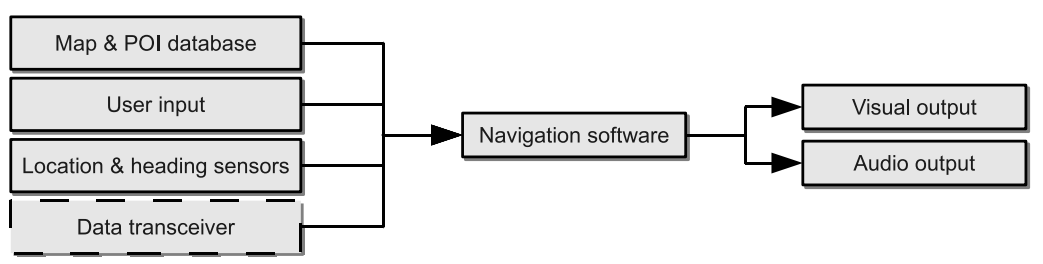

Fig. 1. General architecture of personal navigation systems

\section{State of the Art}

Current personal navigation systems share the common basic architecture depicted in Figure 1 5. The navigation software is responsible for location tracking, route calculation and update toward a destination, and output to the user through both map visualization and voice guidance. Input comes from the following main sources.

1. Map database: which contains geographic and cartographic data, as well as a catalog of POIs. The database can be either pre-loaded and stored locally in the navigation device (such an approach is currently followed in the majority of solutions), or retrieved in real-time during system usage. Hybrid approaches are also possible.

2. User which inserts information about destination, either as address, GPS coordinates or by selecting a POI from the catalog.

3. Location and heading sensors which are key elements in real-time navigation devices. In all current commercial solutions, location is determined through satellite-based technologies, such as GPS (Global Positioning System).

4. Data transceivers, usually based on cellular network connectivity, are currently exploited by many systems to acquire additional information during navigation. This is mainly the case of navigation applications for smartphones, but also several stand-alone device vendors integrate a data module in their highend products. Downloaded data may include temporary map modifications -due to e.g., road maintenance- as well as information about traffic, accidents and weather. That allows the navigation software to determine the best route based not only on static road information, but also on dynamic conditions.

Recent navigation systems provide value-added features for POIs such as phone numbers - directly usable by smartphones and other telephony-enabled navigation devices- and reviews. Nevertheless, significant limitations affect POI management in current solutions: they are organized in a rigid and often oversimplified classification and, as such, they allow category-based or name-based queries only. This prevents users from performing semantically richer searches, limits the quality of resource retrieval and hinders more advanced use cases, such as personalized suggestions based on user profiles. Studies about the behavior of users highlighted the importance of increasing familiarity with the surrounding environment through more sophisticated POI tagging, including social and contextual information [6]. Solutions are thus required for a more expressive 
annotation of POI characteristics. Our proposal supports the above-mentioned features through a robust framework -based on Semantic Web technologies- for resource annotation and discovery, while also tackling practical issues related to the creation, maintenance and storage of world-scale POI catalogs.

A fundamental issue is that cartography databases are proprietary, owned and maintained by companies that license them to navigation system manufacturers. File formats and data structures are not open, hence augmenting data is not possible. On the other hand, creating and maintaining a detailed map database would require huge resource investments, even for a single municipality. Recently, a different approach to map database development was conceived, based on crowd-sourcing: anyone can contribute to a common worldwide cartography by uploading collected data about road segments and POIs to a website. The most mature project is OpenStreetMap: map data are published in an open format under the Creative Commons Attribution-ShareAlike 2.0 licens屯3, granting anyone the permission to use, copy, share and modify. Contributing a road segment to a map region typically involves the following steps.

1. Record a GPS trace while traveling on the road, using GPS logging software which periodically records current geographical coordinates. A collection of data points is obtained.

2. Annotate relevant information about nearby POIs during the trip. This optional step could be performed with paper and pencil, but specialized map collection software has been recently developed for several mobile platforms, integrating a GPS logger and a user interface dedicated to terrain and landmark annotation.

3. Import collected data into an OpenStreetMap-compatible map editor, which reverts sets of raw geographic data points into road segments and allows the user to add road information (such as name, type and speed limit), mark different terrain areas and insert POIs. Finally, collected data are uploaded to OpenStreetMap web servers. After integration into the database, they become visible on the OpenStreetMap website and usable with all compatible software.

A wide range of freely available software tools exists on a wide range of smartphone and computer platforms. In this paper OpenStreetMap is adopted because it proved itself a viable solution for global-scale collaborative cartographic data collection and enrichment, from both legal (licensing) and technical (scalability, interoperability, openness to new features) standpoints.

\section{Framework and Approach}

Main features of the proposed framework are: (i) to follow multi-platform code development rules; (ii) to adopt open source policies in both map data and software components creation; (iii) to enable a semantic-based discovery of POIs

\footnotetext{
${ }^{3}$ Summary and link to full license text are available at http://creativecommons.org/licenses/by-sa/2.0/

4 They are listed in the OpenStreetMap Wiki, http://wiki.openstreetmap.org/
} 


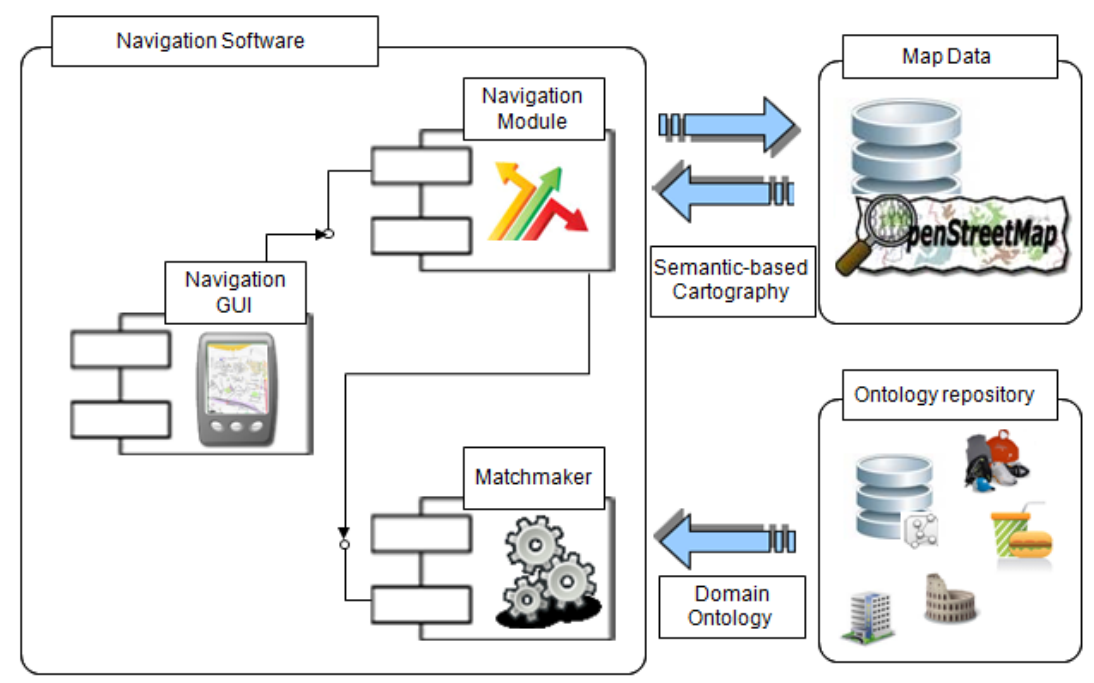

Fig. 2. System Architecture

enhancing the current trivial category-based search of them filtered by nearness criteria. In the following subsections the overall architecture and the map annotation procedure are reported, respectively.

\subsection{System Architecture}

The proposed system adopts a so-called thick client paradigm: differently from classical client-server model, most of the processing is done on the client side. The rationale for this approach is to grant full operation also in case of slow or congested data links. Anyway external interaction is also enabled, via wireless communication protocols, in order to set up further (either client-server or peerto-peer) services. The overall system architecture is depicted in Figure 2,

The mobile client (a standard PDA or smartphone) is equipped with the navigation tool proposed here. Its main components are summarized in what follows. 1. Graphical User Interface (GUI). User can access all functionalities in a quick and straightforward way. Particularly, ontology-based requests addressed to the system can be composed exploiting a graphical toolbar adopting some UI elements familiar to PC users. In Section 4 toy example is presented to better explain how a semantic query is build.

2. Navigation engine. User requests are processed by the navigation module. It extracts information from installed map files and a map graphical view is rendered through the GUI, using the embedded Scalable Vector Graphics engine. We will give a more detailed description of this component later on.

3. Map data files. All map data are locally stored within one or more related files, which encapsulate both geographical data and a semantic annotation of resources. The file structure is described in detail in the next subsection. 
4. Matchmaker. This component is aimed at processing semantic-based user requests exploiting lightweight Concept Abduction, Concept Contraction and Bonus Calculation algorithms. They are used by the navigation module to perform an advanced discovery of the best path for a user also providing a detailed explanation of matchmaking results in order to allow user to refine requests in an iterative way. Semantic Web technologies can provide significant benefits in resource retrieval problems [3]. W.r.t. standard Information Retrieval process, based on keyword search over semi-structured data, a semantic-based matchmaking: (i) overcomes the low recall because of the Open World Assumption paradigm, i.e., a resource is excluded only if its description explicitly contradicts some axioms in the user request; (ii) provides logic-based ranking criteria to select the best matching resources from the available ones; (iii) allows an accurate logic-based explanation of outcomes thanks to the inferred knowledge.

As far as map navigation module is concerned, we decided to extend the Navit5 software navigation tool which is released as open source project under the GPL General Public License. It provides routes calculation and POI finding (but only via a category-based search and selection). Another noticeable feature is multiplatform portability over several mobile Operating Systems through available cross-compilers.

In order to enable semantic-based POI discovery functionalities, the original Navit codebase has been enriched with new software components whose features are explained hereafter while describing operation steps involving the proposed system:

1. User starts navigation tool, selecting the proper application icon on the screen. The navigation module is loaded; it reads a configuration file containing startup information and an annotated user profile expressed in DIG 2.0 [2], a more compact OWL-DL variant. It resumes personal information like interests and hobbies which could either be inserted by the user in a first-time setup phase or imported from other installed application or social network such as Facebook6 and LinkedIn7.

2. The navigation tool checks the matchmaker which runs in background and acts as a server listening for requests submitted by the navigation module (which acts as client).

3. The system starts keeping track of user position, by means of available location services, e.g., internal/external GPS antennas or location APIs.

4. POI discovery can happen in two different ways. (i) User explicitly submits her request selecting related features from main menu. Basically she indicates a general category search (e.g., Cultural Heritage, Accommodation, Entertainment, Dining), each of them corresponding to a given ontology. Then she browses all concepts and roles within the ontology so detailing her request. (ii) Alternatively, the navigation tool is able to retrieve nearby POIs best matching user interests, by simply referring to her profiles and preferences.

\footnotetext{
${ }^{5}$ http://www.navit-project.org/

${ }^{6}$ http://www.facebook.com/

7 http://www.linkedin.com/
} 
In order to take geographical distance into account to grade semantic matchmaking results, we introduce a Score Combination Function (SCF), also known as utility function:

$$
\left.S C F(r, P O I)=100\left[1-\frac{\text { rankPotential }(r, P O I)_{\text {rankPotential }(r, T)}}{\text { rastance }\left(U_{\text {ser_GPS,POI_GPS })}\right.}\right)\right]
$$

where rankPotential $(r, s)$ 3] is the semantic distance measuring the degree of correspondence between request $r$ and point of interest annotation POI, computed solving the Concept Abduction Problem (CAP) [3] rankPotential $(s, \top$ ) is the maximum semantic distance, which depends only on the request and the axioms in the selected ontology. The second local score factor weights the influence of geographical distance: a ratio is used between POI-user distance and maximum distance the user can tolerate for discovery.

\subsection{Map Enhancement}

As said the proposed system is based on OSM. Map data are exported and edited in an XML format adhering to a simple schema which includes three basic elements: (i) nodes, i.e., single geospatial points; (ii) ways, intended as ordered sequences of nodes; (iii) relations, which group multiple nodes and/or ways. Each element can include a given number of general tags having a key/value pair structure. There are no restrictions about attributes, i.e., user can create new needed tags accommodating previously unforeseen uses of maps. With reference to POI description, OSM default representation is made by unconnected nodes, adding one or more additional key-value pairs tag. For instance the amenity=place_of_worship, religion=catholic annotation refers to a catholic church.

In order to enable novel semantic-based discovery of POIs, we have integrated an ontology-based annotation within current descriptions in OSM. Knowledge Representation formalisms have been used, and particularly DIG in a subset of OWL-DL corresponding to the $\mathcal{A L N}$ (Attributive Language with Unqualified Number Restrictions) DL. An important goal of this work is to introduce semantic POI annotation in a way that best fits OSM storage infrastructure. Similarly, mobile computing limitations must be taken into account, and particularly the size of map data to be transferred from OSM server to PDA should be minimized. Therefore, due to the verbosity of XML-based ontological languages such as DIG and OWL, compression techniques [8] have been employed to make semantic metadata more compact.

In detail, two new tags have been introduced in OSM:

$$
\text { <tag k="semantic:ouuid_counter" v="DIG description" /> }
$$

whose key is the OUUID (Ontology Universally Unique IDentifier) code marking the domain ontology the description refers to [7]. The value field contains a semantically annotated DIG description. The counter suffix has been added to allow splitting DIG compressed description in two or more tags. All of them will have the same key value, but a progressively increased counter. 


\section{<tag k="ontology:ouuid" v="Ontology URI" />}

whose value attribute contains the URI from where the ontology can be downloaded. This tag can also be omitted, and in that case the ontology will be searched and retrieved locally from the device storage.

The following three cases are possible: (i) the POI is described using only one ontology and the compressed annotation is shorter than 255 characters; (ii) the POI refers to a single ontology, but compressed description is longer than 255 characters; (iii) the POI is expressed w.r.t. more ontologies. For each of them, the compression procedure accepts in input a DIG/OWL annotation and generates a Base64 string output. If it is shorter than 255 characters, it can be inserted within a single semantic tag, otherwise it is split in 255 characters segments and corresponding tags are inserted within consecutive node elements.

The OSM file including semantic annotations can be finally submitted to the OpenStreetMap server. Then the navigation system will use binary map files generated by the encoding tool, which are smaller than original textual ones. It directly extracts POI annotations from binary data, decodes the compressed description and joins all segments (in case they overcome 255 characters limit).

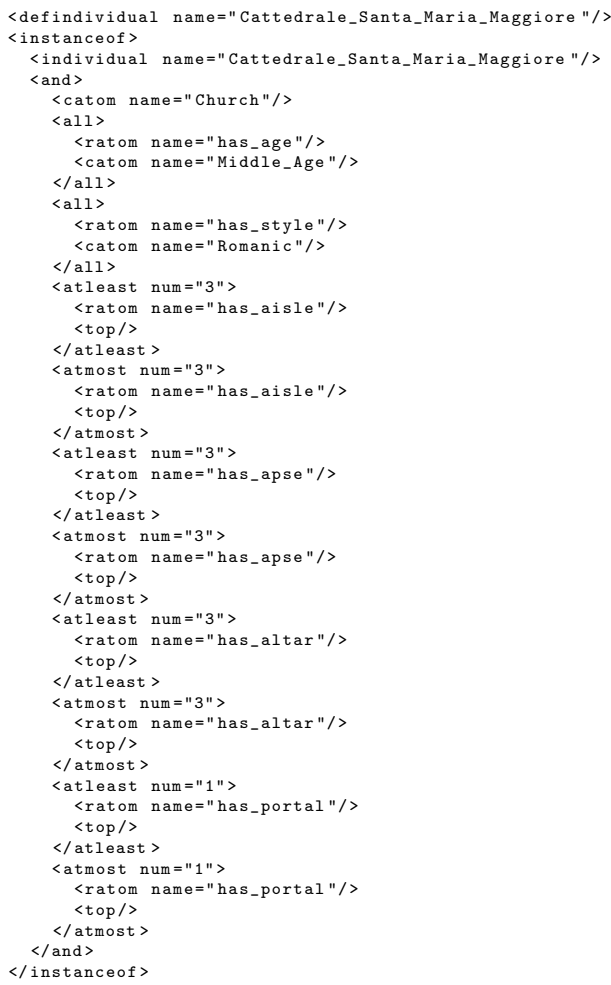

Listing 1. POI DIG description

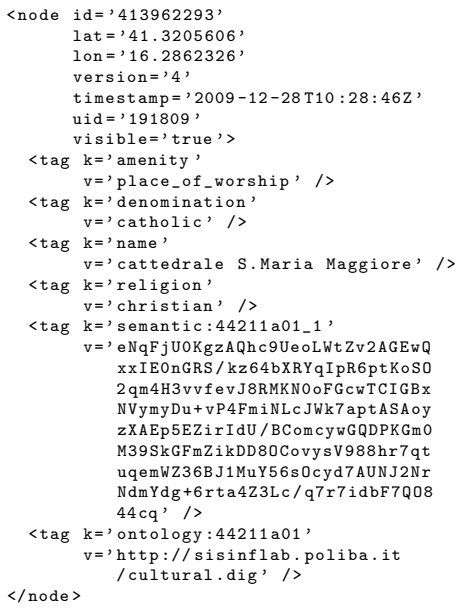

Listing 2. OSM XML excerpt of a semantically annotated POI 
Furthermore, the system checks if the reference ontology is locally available, and if not it is downloaded from the given URI. Finally, a request for matchmaking is addressed to the inference engine running in background.

A basic example of a DIG description is shown in Listing 1, referred to the $S$. Maria Maggiore cathedral in Barletta. In addition, Listing 2 reports on an OSM excerpt of the same semantically annotated POI where semantic annotation has been compressed. It is important to remark that in these examples we used DIG because the matchmaker 4] embedded in our current prototype only supports that interface. However, the proposed approach is general and can work with any other Semantic Web language. A relevant objective for future developments of the prototype is the migration of the matchmaker component to a mobileoptimized inference engine, supporting the most recent standards: OWL 2 as ontology language and OWLlink 8 as reasoner interface.

\section{Case Study: Where Are You Going?}

The extended Navit navigation system was tested in a case study concerning discovery of cultural heritage resources in the Apulia region. Hereafter implementation details are briefly reported and a simple system usage example is described, in order to highlight main features of the proposed approach.

Resource annotation. Map data were collected and added to OpenStreetMap for an uncovered portion of the city of Barletta. An $\mathcal{A L N}$ ontology for description of cultural heritage resources -not reported here due to space constraints- was developed. Several POIs in the Apulia region were annotated w.r.t. it, with highest concentration in Barletta.

Navit evolution. Support for semantically annotated POIs (see Section 3.2) and semantic-based discovery was added to Navit. A very basic cross-platform GUI was developed for ontology browsing and semantic request composition: its purpose was only to allow testing of the solution, while a more sophisticated and user-friendly interface is planned for subsequent system releases. Microsoft Windows Mobile was selected as reference platform for evaluation. MaMaS-tng [4] matchmaker was ported to Windows Mobile and run as a server process. The Navit process acts as a client, interfacing to the inference engine via a local socket using the DIG interface.

Semantic-enhanced POI search. Isabel is in Barletta for the first time, and she would like to visit interesting places. She is particularly fond of modern artistic palaces. She launches Navit on her smartphone and starts a new semantic-based POI discovery, selecting "cultural heritage" as resource domain. The user can browse ontology classes and properties in a dynamically-generated hierarchical GUI structure. She is not only able to choose from a taxonomy of landmark types (see Figure 3(a), 3(b) and 3(c) like in traditional navigation

${ }^{8}$ OWLlink: Structural Specification, Version 1.0, Working Group Recommendation, 16 November 2009, http://www.owllink.org/owllink-20091116/ 


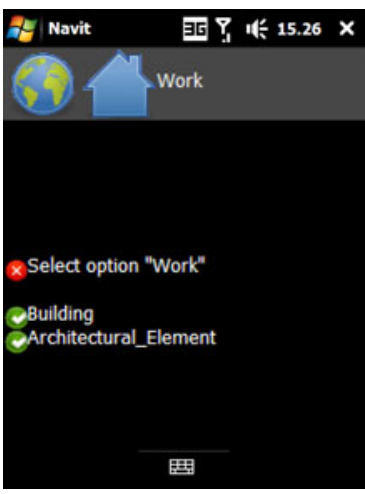

(a)

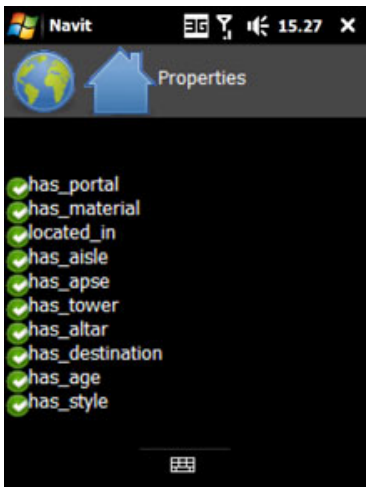

(d)

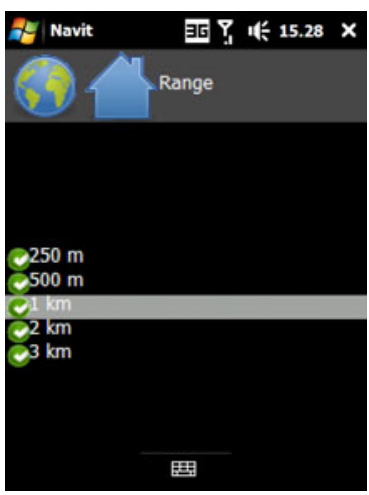

(g)

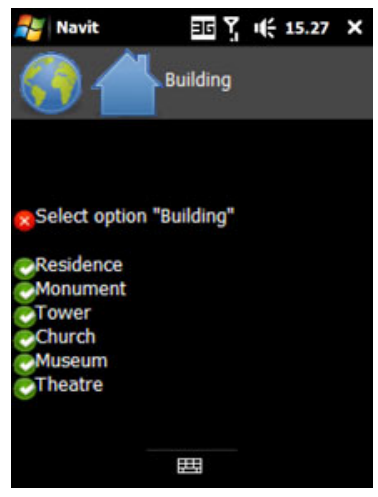

(b)

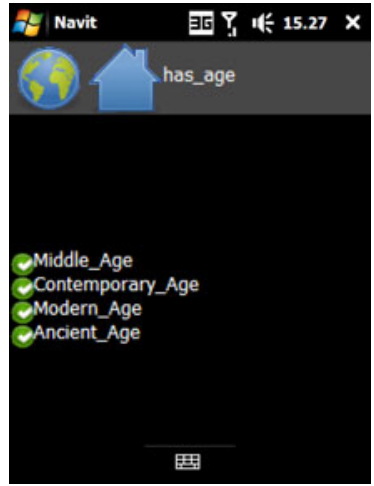

(e)

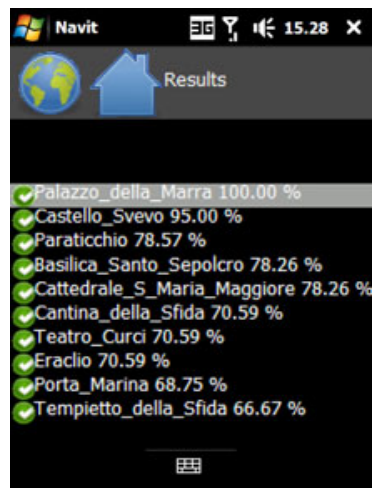

(h)

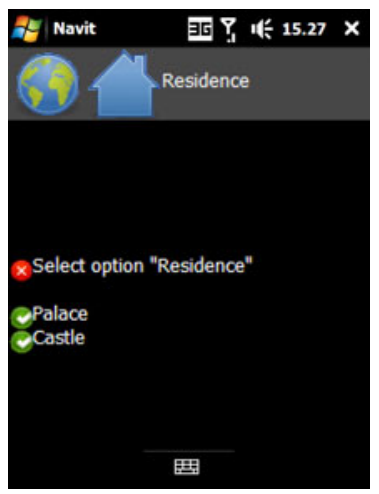

(c)

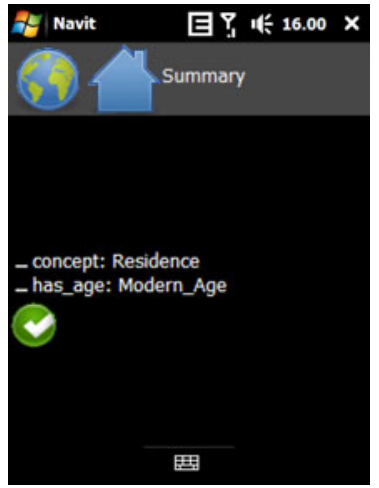

(f)

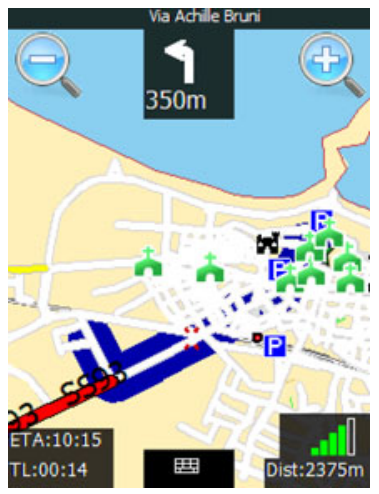

(i)

Fig. 3. Using the semantic-enhanced navigation system 
systems, but also to express the desired resource properties (Figure $3(\mathrm{~d})$ in order to make her search more meaningful and accurate. In our example, after selecting the Palace POI class, she sets her constraint on monument age by navigating the appropriate portion of the ontology model (Figure 3(e)]. In our very short example, the user ignores further available properties, such as style, materials and size. The final semantic request is summarized in Figure 3(f) and corresponds to the expression (in DL notation for the sake of conciseness): Residence $\sqcap \exists$ has_age $\sqcap \forall$ has_age.Modern_Age. The user can review and eventually confirm it. In addition to the semantically annotated expression, the user can insert a maximum acceptable resource distance from her location, as reported in Figure $3(\mathrm{~g})$.

Results review and selection. A Knowledge Base is instantiated in the embedded semantic matchmaker. It consists of the reference domain ontology, of the user request and of POI annotations in the search range. Now inference services for semantic matchmaking are applied. After receiving the reply of the matchmaker, the navigation system computes the utility function, as explained in Section 3.1. In our example, Figure 3(g) reports the final outcome. Only one POI in Barletta, Palazzo della Marra, is a full match for the user request, because it is described as a modern age palace - which is a subclass of residence in the reference ontology- in Baroque style with one portal: Palace $\sqcap \exists$ has_style $\Pi$ $\forall$ has_style.Baroque $\sqcap \quad \exists$ has_age $\Pi \forall$ has_age.Modern_Age $\Pi=1$ has_portal. The next best matching options are Castello Svevo and Paraticchio, which are a medieval castle and a medieval tower, respectively. Their good score is due to the fact that palaces and forts are sibling concepts in the reference ontology. Churches and museums, on the other hand, have higher semantic distance, because of their different nature and purpose. Isabel selects Palazzo della Marra from the result list and navigation begins. Figure 3(i) shows the planned route to the destination; the other POIs in the result list are also marked on the map for further reference.

\section{Conclusion}

We have presented a novel semantic-based navigation approach enabling knowledge-oriented path calculation, which takes into account needs and profile of a user planning a travel. The system we proposed is fully based on open source maps referred to the OpenStreetMap project, which have been semantically enriched to make possible advanced matchmaking services.

Improvements are planned for the embedded matchmaker in terms of performance and support to latest Semantic Web standards. Future work will also aim at extending the proposed system with further vehicle and road information retrieval, including traffic and weather conditions detected by means of Wireless Sensor Networks and OBD-II On-Board Diagnostics system interfaces. Semantic-based map annotation will be facilitated by integrating an ontology-based editor within the OpenStreetMap one. Finally, a throughout experimentation will be carried out in order to improve GUIs and navigation experience recalling user's feedbacks. 


\section{Acknowledgments}

The authors acknowledge partial support of Apulia Region Strategic Project PS 025 - Processes and technologies supporting quasi-markets in logistics.

\section{References}

1. Baader, F., Calvanese, D., Mc Guinness, D., Nardi, D., Patel-Schneider, P.: The Description Logic Handbook. Cambridge University Press, Cambridge (2002)

2. Bechhofer, S., Möller, R., Crowther, P.: The DIG Description Logic Interface. In: Proceedings of the 16th International Workshop on Description Logics (DL 2003), Rome, Italy, September 2003. CEUR Workshop Proceedings, vol. 81 (2003)

3. Colucci, S., Di Noia, T., Pinto, A., Ragone, A., Ruta, M., Tinelli, E.: A nonmonotonic approach to semantic matchmaking and request refinement in emarketplaces. International Journal of Electronic Commerce 12(2), 127-154 (2007)

4. Di Noia, T., Di Sciascio, E., Donini, F.M., Mongiello, M.: A System for Principled Matchmaking in an Electronic Marketplace. International Journal of Electronic Commerce 8(4), 9-37 (2004)

5. French, R.: Maps on Wheels - The Evolution of Automobile Navigation. In: Akerman, J. (ed.) Cartographies of Travel and Navigation, pp. 260-290. The University of Chicago Press, Chicago (2006)

6. Leshed, G., Velden, T., Rieger, O., Kot, B., Sengers, P.: In-car gps navigation: engagement with and disengagement from the environment. In: Proceedings of the Twenty-sixth Annual SIGCHI Conference on Human Factors in Computing Systems, pp. 1675-1684. ACM, New York (2008)

7. Ruta, M., Di Noia, T., Di Sciascio, E., Donini, F.M.: Semantic based collaborative p2p in ubiquitous computing. Web Intelligence and Agent Systems 5(4), 375-391 (2007)

8. Scioscia, F., Ruta, M.: Building a Semantic Web of Things: issues and perspectives in information compression. In: Semantic Web Information Management (SWIM 2009), Proceedings of the 3rd IEEE International Conference on Semantic Computing (ICSC 2009), pp. 589-594. IEEE Computer Society, Los Alamitos (2009) 